\title{
Claudel et la rhétorique de la haine
}

\author{
Glenn Fetzer \\ Calvin College
}

$\mathbf{E}_{\text {n constatant que les « } 2 \text { grandes haines q[u'il a] toujours }}$ gardées au cour sont le protestantisme et l'université ${ }^{1}$ ", Paul Claudel implique autant l'expression rhétorique de ses rancours que la volonté de désigner les objets de sa haine. Au-delà du récit de ces haines, rapports complexes dont de nombreux indices seront lus dans le journal de Claudel et dans ses correspondances, il s'agit dans cette communication non seulement de passer en revue les objets divers de son antipathie mais, surtout, de s'interroger sur la langue et les stratégies stylistiques de ses aversions.

Puisque Claudel se sert lui-même du mot « haine ", il vaut mieux considérer les sens que ce terme peut apporter à la mise en œuvre de l'expression rhétorique. Selon Le Grand Robert de la langue française, le mot « haine " a deux sens : le premier insiste sur la notion de la force du sentiment qui porte à souhaiter du mal à quelqu'un, le second insiste sur celle de la répugnance extrême pour un autre. Le lien est chaque fois la notion des sentiments vivaces et tenaces, et la présence nécessaire d'un objet de ces sentiments. Les deux aspects (la force du sentiment ainsi que son objet) constituent une action ou un comportement qui se rapporte à la rhétorique, surtout dans sa dimension de persuasion, qui, selon Aristote, « est produite par la disposition des auditeurs, quand le discours les amène à éprouver une passion ${ }^{2} »$. Si la persuasion vise les autres, la rhétorique - comme Georges Molinié le précise engage les autres selon ses trois genres principaux : le judiciaire ${ }^{3}$, le délibératif ${ }^{4}$, et le démonstratif. C'est ce troisième trait de la rhétorique - le démonstratif - celui par qui on « peut être amené à apprécier la valeur morale, de bien ou de mal, d'un comportement dans la société ${ }^{5}$ » que Claudel semble suivre dans sa remarque sur ses deux grandes haines. 
Dans son examen rhétorique des passions, Aristote juxtapose la haine à son opposé, l'amitié, qui se fait connaître par son désir du bonheur d'autrui. La haine, par contre, " est engendrée par la colère, par l'outrage et par la calomnie ${ }^{6}$ », et se distingue, d'ailleurs, de la colère, par son objet. Peu semblable à la colère, la haine peut avoir « une raison [...] impersonnelle ${ }^{7}$ ». C'est ainsi que l'on peut comprendre l'invective de Claudel contre le protestantisme et contre l'université : la haine vise des individus, bien sûr, mais aussi des entités et des groupes. Georges Molinié contraste davantage la colère et la haine en commentant la fin de chacune : « en colère », dit-il, « on veut que celui contre qui on en a subisse un mal; quand on hait quelqu'un, on veut sa suppression ${ }^{8}$. » La haine se range, donc, parmi les passions violentes les plus pathétiques; c'est-à-dire, parmi celles comme l'amour, la crainte, la terreur, la pitié, et l'indignation?.

De là vient que la langue peut mettre en relief une rhétorique de la haine. Puisqu'il faut ressentir ces passions autant que possible pour les faire éprouver par l'auditeur, on peut se servir efficacement de l'imagination, des figures, et des lieux ${ }^{10}$. C'est sur cette troisième catégorie - lieux — que Claudel s'appuie pour son expression oppositionnelle.

Le concept de lieu, selon certains rhétoriciens ${ }^{11}$, remonte à la pensée aristotélienne et comprend toutes les formes du raisonnement argumentatif naturel. En répondant aux preuves techniques de l'argumentation et à la matière de l'invention, le lieu peut être classé comme un "stéréotype logico-discursif ${ }^{12}$ ». Autrement dit, c'est ce « sur quoi se rencontrent un grand nombre de raisonnements oratoires ${ }^{13} »$.

Dans le développement de la rhétorique et dans le domaine des belles-lettres (plutôt que dans l'argumentation), on a recours à d'autres configurations, surtout celle des figures, qui comprennent les lieux. Les figures, à leur tour, se scindent selon deux grands types : des figures microstructurales (celles qui se comprennent assez spontanément) et les figures macrostructurales (celles « dont l'existence n'est ni manifeste ni toujours matériellement isolable $\left.{ }^{14} »\right)$. Sous la catégorie " figures macrostructurales de second niveau » 
que Molinié propose pour intégrer les lieux dans les figures, il est possible d'en repérer quelques-unes chez Claudel qui témoignent de la force stylistique de la haine.

En premier lieu le discours de Claudel frappe par le nombre de ses adynatons. Cette figure macrostructurale de second niveau est constituée à la fois d'hyperbole et d'apodioxe (où, pour établir le caractère absurde d'une position contraire, on se sert de l'exagération ${ }^{15}$ ), et se retrouve à plusieurs reprises dans son journal intime. Si Claudel lui-même désigne l'université et le protestantisme comme étant ses plus grandes haines, ce sont ces sujets-là que les adynatons illustrent parfois.

« Ce que je ne puis pardonner à Napoléon, c'est institution de l'université, le plus bel instrument d'abrutissement qui ait jamais été conçu par un tyran ${ }^{16}$. »

Cette remarque de Claudel illustre l'utilisation de l'adynaton dans la mesure où elle fait usage d'apodioxe, en ce qu'elle $1^{\circ}$ ) évoque un adversaire [Napoléon], et $2^{\circ}$ ) lui attribue une position quelconque [l'établissement de l'université] que Claudel rejette comme évidemment nuisible et, par conséquent, sans valeur. En même temps la remarque s'exprime en tant qu'hyperbole, dont le côté exagéré est manifeste et par le vocabulaire fortement intensif (les noms généraux « abrutissement ", et « tyran », par exemple) et par le subjonctif qui cerne un état de choses non concrétisées. L'adynaton, dans ce cas, est rendu fort grâce à sa structure d'apposition.

L'université se trouve de nouveau objet de dédain dans le journal de Claudel quelques années plus tard. En 1940, inquiet du sort de son fils Pierre et de l'avenir de la France, Claudel note la remarque suivante : « Prier, prier, prier, et encore prier pour q[ue] Pierre soit sauvé, pour q[ue] les Boches ne reviennent pas, pour q[ue] la France soit sauvée. Spes contre spem! Dans l'Exp[osé] des motifs on reconnaît le mal fait par l'éducation sans Dieu. C'est toute l'Université, œuvre de Napoléon, qu'il faudrait $f$... par terre. Toute l'idolâtrie classique ${ }^{17}$ ». Dans cette occurrence de l'adynaton, on se rend compte de la présence d'une structure semblable à celle 
de mon premier exemple. En désignant le responsable du mal ( « l'adversaire », qui, encore une fois, est Napoléon), il cerne l'argument (ou le produit de Napoléon — dans ce cas, l'université), qu'il faut - à son avis - supprimer. Semblable à la figure considérée précédemment, toute l'entité dont l'auteur veut souligner l'absurdité, est présentée d'une manière concise, et cela, par l'usage de l'apposition, dont la force vient de l'expression totalisante de l'hyperbole (« toute l'idolâtrie classique »). Dans l'un et l'autre cas, l'usage de l'adynaton nous mène à observer une structure particulière dans les journaux, une structure qui ne se borne pas à l'adynaton mais qui est celle d'une relation appositive, où un énoncé elliptique et concis sert de proposition explicative ${ }^{18}$.

Malgré la présence de l'adynaton dans le journal intime, c'est avec des essais tels que "Réflexions et propositions sur le vers français » que l'on trouve des figures dans un contexte élaboré en vue de faciliter la persuasion. Dans le passage où Claudel, dans une tentative d'illustrer la force de la prosodie française, se propose de résumer la psychologie du peuple français, on observe une suite de figures macrostructurales de second niveau, toutes engagées en quelque sorte, dans une rhétorique oppositionnelle. Une occurrence de l'adynaton sert à renforcer la notion de la parcimonie des Français. La situation décrite est hyperbolique : « Le Français s'est toujours senti actionnaire d'une société dont chaque membre doit des comptes à tous les autres. Il ne veut rien laisser perdre. Un bien inutile et gâché, un agrément qu'on se donne, lui paraissent de mauvaises actions. De là notre renommée de parcimonie et épargne ${ }^{19}$ ". A l'hyperbole s'ajoute l'apodioxe quand Claudel évoque la position d'un tiers (ici, Balzac, et son portrait du Père Grandet avare), pour contester le caractère avare du Père Grandet : « Le Père Grandet qu'a si bien décrit Balzac n'est pas proprement un avare, c'est un homme qui n'est à l'aise que dans la nécessité. » L'apodioxe se trouve, donc au centre de l'adynaton et introduit la position de Claudel : "Cette défiance, du plaisir, même innocent, explique notre morale négative et sèche, notre critique de la vie amère et pessimiste $^{20}$. $\gg$ C'est donc à force d'exagération et de rejet de la position attribuée à un autre que l'essayiste tente de persuader son lecteur. 
A ces adynatons s'ajoutent d'autres figures macrostructurales de second niveau, surtout l'antéoccupation, l'auxèse, et l'éthopée. Lors du développement discursif sur la psychologie des Français, Claudel se sert de l'antéoccupation pour montrer son argument. Cette figure propose le stéréotype d'un tiers pour prendre les devants dans la discussion. Dans l'antéoccupation, " on feint qu'[un] adversaire, ou simplement l'interlocuteur, lui oppose une objection et l'exprime ${ }^{21}$ ». Dans « Réflexions et propositions sur un vers français », on trouve une phrase, située entre deux figures, qu'on peut qualifier d'antéoccupation : "Les jansénistes se défiaient même de l'eucharistie et La Rochefoucauld est comme obsédé par l'idée de l'amour-propre ${ }^{22}$. » Toujours dans le but de persuader son lecteur que tout ce qui n'est pas nécessaire provoque chez le Français un sens profond d'inquiétude, Claudel offre ces deux stéréotypes qui, par leur brièveté et par leur structure de propositions principales successives, accentuent le courant de l'argumentation. D'ailleurs, par le choix d'objet de stéréotypes - les jansénistes et La Rochefoucauld - Claudel fait voir certaines de ses antipathies de longue date.

Chez Claudel la présentation des arguments n'est jamais effectuée uniquement à force de propos sémantiques. On peut donc bien dire avec Laurence Bougault que la figure de pensée « apparaît comme [...] élément perturbateur de la linéarité de la sémantique $^{23} \gg$. C'est ainsi que, par le retour de ces éléments qui troublent la linéarité du texte, une intensité rythmique s'instaure, qui aiguise la mise en épreuve des passions. Outre les figures de l'adynaton et de l'antéoccupation, on rencontre dans l'essai sur le vers français la figure de l'auxèse (procédé par lequel on se sert d'un « enchaînement d'expression hyperbolique, dans une orientation continue et méliorative $\left.{ }^{24} »\right)$. Les traits saillants de cette figure se comprennent comme $1^{\circ}$ ) les liaisons des choses, et $2^{\circ}$ ) la nature hyperbolique de ces éléments.

L'agencement de l'enchaînement dans le texte se fait de façon interne aussi bien qu'externe. A l'intérieur de la figure on trouve une suite d'éléments parallèles qui, par leur similitude, constituent une série verbale. Dans « Réflexions et propositions sur 


\section{Glenn Fetzer}

le vers français » on trouve plusieurs occurrences de ces séries. L'entité à répéter en série peut se constituer de nombreuses structures. On trouve, par exemple, l'assemblage $1^{\circ}$ ) nom, $2^{\circ}$ ) adjectif antéposé, et $3^{\circ}$ ) article anaphorique : « La même horreur du hasard, le même besoin de l'absolu, la même défiance de la sensibilité ", dans l'auxèse qui termine ainsi : « qu'on retrouve encore aujourd'hui dans notre caractère et nos arrangements sociaux, ont modelé notre grammaire et notre prosodie ${ }^{25} »$. Cette auxèse et les deux qui suivent font partie d'une illustration des « commandements de Dieu et de l'Eglise » ainsi que de « la sagesse des villageois » en informant la poésie française classique. Si cette figure en particulier cherche à renforcer le caractère « gnomique et mnémotechnique »de la poésie française classique, elle le fait à force d'accentuer les extrêmes.

Un autre paradigme s'esquisse dans l'auxèse suivante : un paradigme régi par le verbe de modalité «falloir ».

«Il fallait empêcher l'air d'entrer, il fallait exclure toute espèce de jeu et de décalage, il fallait serrer les mots par une contrainte extérieure et intérieure si forte, entre des coins si durs, que la ligne acquît l'immobilité définitive et infrangible d'une inscription, lisible pour l'éternité ${ }^{26}$. "

D'après Harald Weinrich, le but de ce trait verbal est d'inciter le lecteur à devenir le sujet d'action ${ }^{27}$. Dans ces propos, l'expression oppositionnelle s'annonce avec force. Non seulement aspire-t-on à l'évitement (sinon suppression) d'un état de choses, mais aussi la structure des propos implique le lecteur dans la démarche.

Deux auxèses de plus se suivent d'emblée :

$1^{\circ}$ ) «C'est à quoi l'on parvient non seulement par la rime, par la césure fixe et par la fixation d'un nombre obligatoire de syllabes, mais encore par l'exclusion des muettes intérieures non élidées (comme vues et fées), par la proscription de l'accouplement entre les genres et 
les nombres, toutes tortures auxquelles certains zélotes ajoutèrent des mortifications personnelles et surérogatoires, telles que la rime riche, la rime rare et l'abstinence du hiatus. »

$2^{\circ}$ ) «Sans parler du code général de la grammaire et de la rhétorique, du 'savoir-vivre et du goût', plus vétilleux que l'ancienne étiquette espagnole ${ }^{28}$. »

Si l'auxèse se distingue par l'enchaînement d'expressions, la préposition par convient bien à la figure. Weinrich nous rappelle que la préposition par «n'existe que comme forme liée ${ }^{29}$ » et peut signaler passages, distributions, médiations. Bref, en étant composé du trait sémantique d'élargissement, par illustre, en quelque sorte, le sens de l'auxèse. Bien entendu, on n'ose pas prétendre que la haine se dégage de chacun de ces exemples de l'auxèse. Nous nous rappelons que cette figure ne marque qu'une occasion digressive lors d'une stratégie de traiter globalement un thème. Cependant, par la mise en jeu des expressions exagérées, l'auxèse s'harmonise avec la force du sentiment et l'aversion pour un tiers que présente la haine.

Pour reprendre la notion d'enchaînement dans le texte dont témoigne l'auxèse, les usages successifs de l'auxèse démontrent que la perturbation de la linéarité de la sémantique peut se frayer en toute intensité. La contiguité de la figure de l'auxèse nous rappelle que, selon Gilles Deleuze, « la répétition est la puissance du langage ${ }^{30} » . E t$, poursuit-il, « loin de s'expliquer de manière négative, par un défaut des concepts nominaux, elle [c'est-à-dire la répétition] implique une idée [...] excessive ${ }^{31}$. »

$\mathrm{Si}$ la rhétorique de la haine doit son inscription - en partie - à la vivacité et la ténacité de la parole, dans « Réflexions et propositions sur le vers français » l'expression de cette rhétorique se fond sur un rapport quelconque avec un individu ou personnage. Puisque dans son journal intime Claudel n'hésite pas à commenter l'aspect moral, psychologique, ou physique de quelqu'un, il n'est pas surprenant de trouver des descriptions des gens dans l'essai sur le vers français. En effet, au moins deux figures de ce genre s'y trouvent : l'éthopée et la prosographie. La première vise la description 


\section{Glenn Fetzer}

morale et psychologique d'une autre personne, « de manière à ce que tout le développement du discours soit commandé par ce traitement $^{32}$ », et la deuxième s'attache plutôt à l'aspect physique ${ }^{33}$.

L'usage de ces deux figures se trouve surtout dans la « Digression sur Victor Hugo », où l'essayiste, lors d'un hiatus dans le développement de la discussion sur la versification, parle du marbre de Hugo qui est au Musée de Copenhague. L'intérêt de Claudel pour la description physique d'Hugo se lit dans les lignes suivantes, qui servent d'introduction aux propos de Claudel :
« [...] un grand front n'exprime pas toujours l'intelli- gence, mais bien des fois simplement la vacance et l'évaporation. Un nez rond ne signifie pas toujours la bonté. Un menton en galoche n'est pas toujours un indice de caractère, le museau de carpe de la Reine Victoria par exemple n'est pas dénué d'une énergie qui va jusqu'à la férocité, il a le sérieux qui manque à beau- coup de casse-noisettes. Aucun trait de la figure ne peut s'interpréter isolément comme un article de vocabulaire. Il faut se rapporter au contexte tout entier et non seule- ment à la figure, mais au corps même que la tête récapitule, à ses attitudes, ses mouvements, ses aplombes, sa taille, son volume ${ }^{34}$.»

Bien que ces lignes ne se rapportent pas ouvertement à un personnage spécifique, elles préparent une analyse à venir. Ainsi, elles annoncent un élément constitutif d'une figure plus globale, qui est le portrait. Après encore un autre paragraphe qui prolonge cette stratégie, Claudel entreprend sa description du marbre de Victor Hugo.

Il part de l'aspect physique, bien sûr, mais selon les traits qu'il vient de présenter, la description tourne bientôt en commentaire sur la morale et sur la psychologique :

«Le marbre de Victor Hugo au fond de ce sombre Musée de Copenhague, auquel me ramenait toute une espèce de 
fascination, fournissait un magnifique exemple de cette architecture spirituelle. [...] Sous ce front olympien la figure moyenne fait un étrange contraste. Je parlerai des yeux tout à l'heure. Mais tout l'ensemble de la figure n'avance pas, il oppose à la vie, sauf la courte saillie du nez, une élévation verticale. Et ce nez même ne respire pas; sur certains portraits ce n'est qu'un trognon sensuel, sur le buste de Rodin, c'est le bec recourbé, dur et court, d'un petit rapace, émouchet ou buse. Le tout sous le poids énorme du front a je ne sais quoi de tassé, de comprimé, de colérique, de bouché, de mesquin, d'hostile et de méchant. - Que l'on compare cette physionomie maçonnée avec celle d'un intérieur, d'un pur pneumatique, Baudelaire, par exemple, ou Dalou, dont un autre buste à côté dessine le long nez délicat et ciselé. [...] Mais les images antérieures à 1861 montrent une large bouche aux bords minces d'acteur ou d'avocat soutenu par cette lèvre inférieure fine et bien détachée qui permet aux Français, m'a dit un musicien, d'être les meilleurs joueurs de clarinette de l'univers. Les paroles doivent se détacher comme des pièces d'or de ce trébuchet excellent. A l'ensemble sert de base la volute assez courte, mais nettement accentuée d'un menton égoïste et affirmatif. La tendresse, la bonté, la sympathie, et surtout la joie, ne rayonnent pas précisément de cette physionomie imposante et sinistre ${ }^{35}$. "

En physionomiste, Claudel suggère le caractère de l'individu représenté par le marbre. On remarque pourtant deux aspects de l'extrait qui frappent le lecteur : $1^{\circ}$ ) la nature psychologique s'esquisse, en partie, par des comparaisons avec d'autres marbres ceux de Rodin, de Baudelaire, et de Dalou. Cette appréciation empruntée à la physionomie rappelle - si seulement de loin - le lieu de l'apodioxe. $2^{\circ}$ ) l'essayiste profite de l'imprécision pour monter à l'assaut contre l'adversaire. De ce manque d'exactitude résultent deux élans expansifs. Le premier se voit à force d'ep- 
ithètes dont les sens s'avèrent de plus en plus défavorables ( le tout [...] a je ne sais quoi de tassé, de comprimé, de colérique, de bouché, de mesquin, d'hostile et de méchant »), et le deuxième, composé de noms communs, sert à résumer tout l'argument ( La tendresse, la bonté, la sympathie, et surtout la joie, ne rayonnent pas... »).

L'extrait propose en effet une éthopée qui se joue au niveau d'un discours moral. Cette figure est produite ici par la métamorphose des traits physiques clichés -signalés tôt dans le passage par la prosopographie - en valeurs morales. Si Claudel s'exerce longuement à présenter une description péjorative de Victor Hugo (ou, plutôt, de son marbre), ce n'est pas pour s'attarder sur l'homme. La digression sur Victor Hugo finit par viser l'œuvre d'Hugo, à laquelle Claudel s'oppose vivement. Un exemple bref suffira afin de faire voir son antipathie pour l'œuvre d'Hugo :
«On peut dire sans exagération que le sentiment le plus habituel à Victor Hugo, celui où il a trouvé ses inspira- tions les plus pathétique, celui auquel il n'a jamais recours en vain et qui lui fournit un répertoire inépuis- able de formes et de mouvements, sa chambre intérieure de torture et de création, c'est l'épouvante, une espèce de contemplation panique ${ }^{36}$. "

L'usage de la prosopographie et de l'éthopée comme éléments constitutifs d'un développement plus global illustre bien la stratégie de la part de l'auteur d'infléchir par réaction l'opinion du lecteur.

Si l'on trouve la plupart des expressions de l'antipathie claudelienne dans le Journal, les correspondances, et les essais, les poèmes n'en sont pas non plus exclus. Parmi les passages de poésie où l'on voit l'imbrication de plusieurs figures, notamment l'antéoccupation, l'apodioxe, et l'adynaton, l'extrait suivant, du Magnificat, du Troisième Ode, est à l'œuvre :

Puisque comprendre, c'est refaire

La chose même que l'on a prise avec soi. 


\section{Claudel et la rhétorique de la haine}

Restez avec moi, Seigneur, parce que le soir approche et ne m'abandonnez pas!

$\mathrm{Ne}$ me perdez point avec les Voltaire, et les Renan, et les Michelet, et les Hugo, et tous les autres infâmes!

Leur âme est avec les chiens morts, leurs livres sont joints au fumier.

Ils sont morts, et leur nom même après leur mort est un poison et une pourriture.

Parce que vous avez dispersé les orgueilleux et ils ne peuvent être ensemble,

$\mathrm{Ni}$ comprendre, mais seulement détruire et dissiper, et mettre les choses ensemble.

Laissez-moi voir et entendre toutes choses avec la parole

Et saluez chacune par son nom même avec la parole qui l'a fait ${ }^{37}$.

Malgré la mise en avant des figures considérées, le côté métaphorique du poème domine la lecture. L'usage des noms propres - Voltaire, Renan, Michelet, Hugo - en tant qu'objet de dédain et, même, d'aversion - fait référence aux contextes historiques et culturels. Derrière chaque nom propre il reste une archéologie. Les quatre noms découvrent de vieux débats en même temps qu'ils se donnent pour la construction du poème. Ils renvoient avant tout aux valeurs morales et religieuses qui caractérisent l'œuvre claudelienne.

Loin d'apparaître comme un phénomène arbitraire, la rhétorique de la haine, au regard des quelques analyses qui précèdent, nous semble faire partie de l'écriture oppositionnelle chez Claudel. On pourrait étudier beaucoup d'autres exemples de cette rhétorique - non seulement les nombreux cas dans les Journaux, mais également dans les essais et les discours (on pense surtout au «Discours sur les lettres françaises " [1922], à la " Réponse à une enquête sur la littérature » [1940], au " Discours de clôture à la réunion des publicistes chrétiens " [1946], et ainsi de suite). En fin de 


\section{Glenn Fetzer}

compte, l'écriture oppositionnelle renvoie au domaine de l'esthétique et démontre que chez Claudel, l'expression de l'esprit critique n'est jamais obscurcie ni n'est jamais indifférente aux circonstances de son époque.

\section{Notes}

1 Paul Claudel, Journal II (1933-1955), Paris : Gallimard, 1969, p. 89. La remarque a été écrite en avril 1935.

2 Aristote, Rhétorique, 1355b-1356a, trad. M. Dufour, Pars : Les Belles Lettres, 1967, p. 76.

3 Selon Molinié, par le judiciaire, on est «amené à tenter de faire trancher une question par oui ou par non» (voir Michèle Aquien et Georges Molinié, Dictionnaire de la rhétorique et de poétique La Pochotèque, Livre de Poche, 1999, p. 8).

4 Par le délibératif, Molinié entend qu'on est «amené à faire s'interroger sur l'opportunité d'une décision» (Dictionnaire de la rhétorique et de poétique, p. 8).

5 Dictionnaire de la rhétorique et de poétique, p. 8.

6 Dictionnaire de la rhétorique et de poétique, p. 299.

7 Dictionnaire de la rhétorique et de poétique, p. 299.

8 Dictionnaire de la rhétorique et de poétique, p. 299.

9 Dictionnaire de la rhétorique et de poétique, p. 292.

10 Dictionnaire de la rhétorique et de poétique p. 299.

11 Georges Molinié détaille ce concept sous la rubrique "lieu" (Dictionnaire de la rhétorique et de poétique p. 224).

12 Dictionnaire de la rhétorique et de poétique, p. 223.

13 Dictionnaire de la rhétorique et de poétique, p. 224.

14 Dictionnaire de la rhétorique et de poétique, p. 177.

15 Dictionnaire de la rhétorique et de poétique, p. 66.

16 Paul Claudel, Journal II, p. 37. Cette remarque date du 8 sept. 1933. 
17 Paul Claudel, Journal II, p. 322. Cette remarque date du 9 juillet 1940.

18 Voir les remarques suivantes : "L'un de mes fils épouse une Grecque, l'autre une Américaine protestante. L'hérésie et le schisme », Journal II, p. 737 [juillet 1950].

19 Claudel, Positions et propositions, tome Quinzième. Eurres complètes. Paris: Gallimard, 1959, p. 15.

20 Claudel, Positions et propositions, p. 15.

21 Dictionnaire de la rhétorique et de poétique, p. 59.

22 Claudel, Positions et propositions, p. 15.

23 Laurence Bougault, "A propos du rythme en poésie moderne », Revue Romane 342 1999, p. 247.

24 Dictionnaire de la rhétorique et de poétique, p. 59.

25 Claudel, Positions et propositions, p. 16.

26 Claudel, Positions et propositions, p. 16.

27 Harald Weinrich, Grammaire textuelle du français. Paris : Didier/Hatier, 1989, p. 196.

28 Claudel, Positions et propositions, p. 16.

29 Weinrich, p. 438.

30 Gilles Deleuze, Différence et répétition, Paris : PUF, 1968, p. 373.

31 Deleuze, p. 373.

32 Dictionnaire de la rhétorique et de poétique p. 164.

33 Dictionnaire de la rhétorique et de poétique p. 324.

34 Claudel, Positions et propositions, pp. 27-28.

35 Claudel, Positions et propositions, pp. 20-21.

36 Claudel, Positions et propositions, p 30.

37 Paul Claudel, Poésie, tome Premier. CEurres complètes de Paul Claudel.

Paris : Gallimard, 1950, p. 108. 